\title{
Les écoles Montessori dans le monde
}

La diversité interne d'un réseau en expansion

Montessori schools around the world. The internal diversity of an expanding network

Las escuelas Montessori en el mundo. La diversidad interna de una red en plena expansión

Marie-Laure Viaud

\section{OpenEdition}

Journals

Édition électronique

URL : https://journals.openedition.org/ries/6047

DOI : $10.4000 /$ ries.6047

ISSN : 2261-4265

Éditeur

France Education international

Édition imprimée

Date de publication : 1 décembre 2017

Pagination : 51-62

ISBN : 978-2-85420-616-6

ISSN : $1254-4590$

Référence électronique

Marie-Laure Viaud, «Les écoles Montessori dans le monde », Revue internationale d'éducation de Sèvres [En ligne], 76 | décembre 2017, mis en ligne le 01 décembre 2019, consulté le 15 février 2023. URL : http://journals.openedition.org/ries/6047 ; DOI : https://doi.org/10.4000/ries.6047 


\title{
Les écoles Montessori dans le monde
}

\section{La diversité interne d'un réseau en expansion}

\author{
Marie-Laure Viaud \\ Université d'Artois
}

Les écoles Montessori se multiplient dans de très nombreux pays. En France, on dénombrait 125 écoles revendiquant une appellation Montessori en 2012 , et 169 en 2017, soit un taux de progression de plus de $6 \%$ par an ${ }^{1}$. La situation est la même dans de nombreux autres pays, comme le montre l'analyse de la presse (Viaud, 2017) ou les requêtes réalisées avec l'outil Google Trend pour mesurer l'intérêt suscité par le terme "Montessori » auprès des internautes.

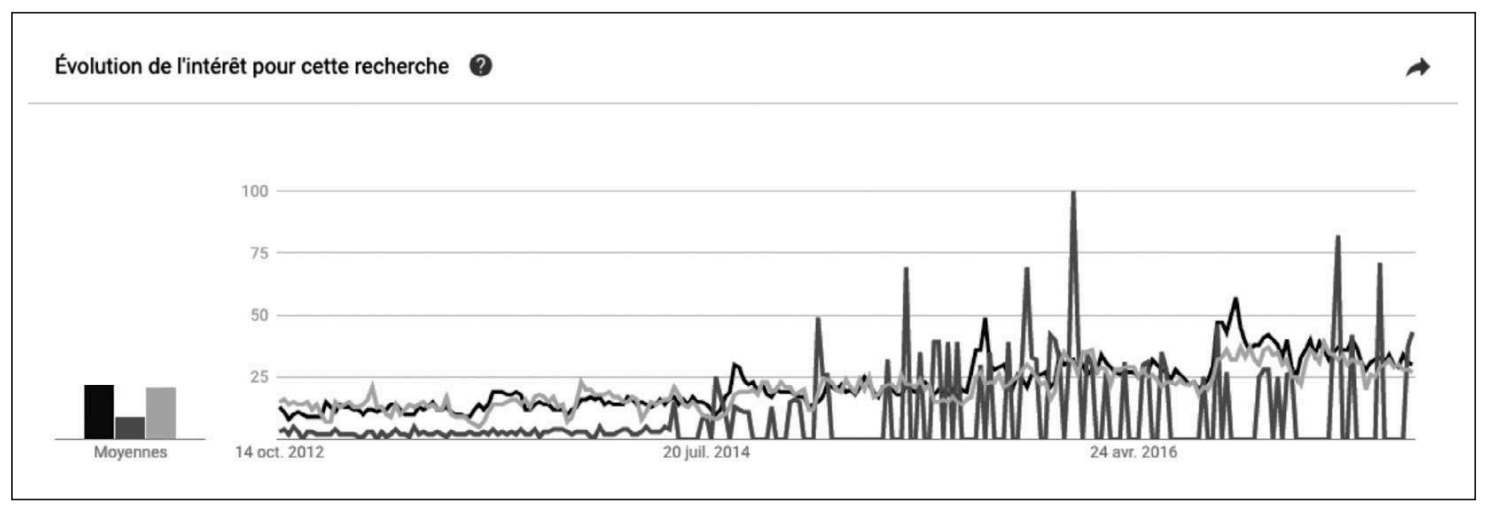

L'utilisation du terme « Montessori » dans le moteur de recherche Google entre 2012 et 2017 en France $(-)$, en Chine $(-)$ et en Espagne $(-)$.

De toutes les pédagogies alternatives issues de l'Éducation nouvelle, c'est la seule à être diffusée sur tous les continents. Une recherche sur Internet à partir des mots clés «nom de capitale + Montessori » montre qu'il existe des jardins d'enfant ou des écoles Montessori dans quasiment toutes les grandes villes du monde : c'est par exemple le cas de 52 des 55 capitales africaines. Il existerait au total près de 22000 écoles dans le monde entier (Verdiani, 2012), comportant

1. Recensements effectués par l'auteure pour les deux éditions de l'ouvrage : Viaud, Montessori, Freinet, Steiner: une école différente pour mon enfant ? Nathan, édition 2012 et édition 2017. 
majoritairement des classes maternelles et élémentaires. En outre, un nombre croissant d'écoles alternatives revendiquant des pratiques pédagogies hybrides utilisent aussi les outils Montessori.

Quelles sont ces écoles Montessori ? Comment expliquer leur développement ? Pourquoi, de toutes les pédagogies nouvelles, la pédagogie Montessori connaît-elle le succès le plus important?

Pour répondre à ces questions, nous avons étudié un panel d'une centaine d'écoles Montessori situées dans différents pays $^{2}$, par le biais des pages Internet ou Facebook où elles se présentent, en comparant la pédagogie, la présentation de leur fonctionnement (locaux, nombre d'élèves, activités, coût, public visé...) et l'argumentaire généralement présent sur une page consacrée aux «missions» ou à la "philosophie» de l'école. Une analyse des photos présentées sur les pages d'accueil des sites de deux à quatre écoles de chaque type a été réalisée en prenant appui sur la sémiologie de la signification qui invite à décoder les signes qui ne sont pas lisibles de prime abord (Barthes, 1964).

Cette étude est restreinte aux seules écoles Montessori et n'aborde donc pas le développement de cette pédagogie dans les classes. Elle est fondée sur l'auto-présentation de ces écoles : certaines des affirmations qui suivent, concernant par exemple le public qui fréquente tel ou tel type de structure, pourraient être nuancées ou invalidées par des études de terrain. Les écoles prises en compte sont celles qui revendiquent l'appellation Montessori, qu'elles appartiennent ou non à l'Association Montessori Internationale (AMI). Enfin, le caractère réduit de cette étude par rapport au nombre total d'écoles Montessori existant dans le monde et l'obligation que nous avons eue de nous limiter aux écoles disposant d'un site en caractères latins (excluant nombre d'écoles russes, chinoises, arabes, etc.) obligent à considérer ce travail comme uniquement exploratoire.

Nous présenterons d'abord une typologie des différents modèles d'écoles Montessori observés, puis une analyse des facteurs pouvant expliquer leur développement.

\section{LA GRANDE DIVERSITÉ DES ÉCOLES MONTESSORI}

De la comparaison des écoles étudiées, il ressort que celles-ci sont d'une grande diversité. Nous avons dégagé cinq types, sachant qu'il s'agit de modèles idéal-typiques (au sens où ceux-ci ont été définis par Max Weber) et que certaines écoles peuvent se trouver au croisement de plusieurs logiques.

\footnotetext{
2. Ces écoles ont été localisées en utilisant, dans un moteur de recherche, les mots clés « Montessori + nom d'une capitale ». Nous avons exploré de façon systématique les réponses obtenues pour l'ensemble des 54 capitales africaines et effectué des sondages plus partiels pour des villes de différentes régions du monde : Paris, New-York, Vancouver, Shanghai, Bangkok, mais aussi Alma-Ata ou Bichkek.
} 


\section{Des écoles élitistes, internationales et bilingues}

Des écoles Montessori élitistes, internationales et bilingues existent dans la majorité des grandes capitales, comme à Paris, avec The Bilingual Montessori School of Paris (BMS), ou au Rwanda où se trouve The international Montessori School of Rwanda (IMS).

La comparaison entre les pages d'accueil des sites de ces deux écoles montre que les photos choisies sont assez semblables : ce sont des images, en plan large, de salles de classes vastes, lumineuses, à la décoration épurée et soignée comme dans un magazine de décoration d'intérieur : soleil qui rentre à flot et illumine les beaux meubles de bois clair, bouquet de fleurs fraîches... et présence d'un seul enfant au travail. En somme, tout dans le cadre annonce l'exception, le calme, la qualité de ce qui est offert ici, et l'attention individuelle dont il est possible de bénéficier.

Le coût est d'environ 14000 euros annuels pour la scolarité primaire, dans les deux écoles citées. Le taux d'encadrement est de l'ordre d'un adulte pour douze élèves. L'enseignement est bilingue : deux langues, dont l'anglais, sont utilisées à part égale dans l'enseignement, à l'oral comme à l'écrit. À côté du traditionnel travail sur le matériel Montessori, les activités sportives et de plein air occupent une place importante : ainsi, l'IMS dispose de sa propre piscine privée et d'équipements hauts de gamme (salle de danse), tandis que les élèves de la BMS participent tous, chaque année, à une semaine de ski en Suisse et à une autre d'équitation.

Ces écoles s'adressent principalement aux enfants d'expatriés et de diplomates: BMS comme IMS reçoivent chacune une vingtaine de nationalités différentes ; l'un des sites parisiens est d'ailleurs installé Quai d'Orsay, à quelques pas du ministère des affaires étrangères. Implantées dans la plupart des grandes capitales du monde, elles se ressemblent beaucoup entre elles, permettant aux enfants de retrouver, au fil de leurs déménagements successifs, les mêmes méthodes, le même matériel, le même aménagement des salles de classe et la même langue de communication.

\section{Des apprentissages précoces au service de l'ascension sociale}

Un deuxième type d'écoles Montessori, qui se retrouve dans de très nombreux pays, s'auto-présentent comme étant destinées à des familles désireuses d'offrir à leurs enfants la meilleure réussite scolaire possible dans une stratégie d'ascension sociale. C'est par exemple le cas de Montessori Academy de Vancouver, au Canada (EMA), de l'Elite Montessori School d'Accra au Ghana (EMS) ou de la Dove Montessori School de Kigali au Rwanda (DMS). 
Ces écoles, qui semblent majoritairement fréquentées par les nouvelles classes moyennes et supérieures locales ${ }^{3}$, mettent l'accent sur les apprentissages plutôt que sur les autres particularités de la pédagogie Montessori. Les photos illustrant les pages d'accueil des sites web sont celles d'enfants appliqués, concentrés sur un matériel d'écriture ou de mathématiques, comme les lettres rugueuses. Dans l'auto-présentation de la DMS, les apprentissages académiques sont les premiers cités :

notre objectif est d'aider chaque enfant à développer son plein potentiel, académiquement, socialement, émotionnellement et physiquement.

Un article d'un magazine local consacré à EMA est explicite :

Voulez-vous avoir un enfant trilingue? Chez Elite Montessori, votre enfant a l'opportunité d'apprendre l'anglais, le français et le mandarin au cours d'une journée. (...) Un élève de quatre ans sait déjà multiplier et peut nommer toutes les planètes ${ }^{4}$.

En revanche, les activités sportives, culturelles et artistiques ne sont pas mises en avant: pour ces familles, elles ne constituent probablement pas un élément prépondérant.

\section{Épanouissement, confiance en soi, solidarité}

Ce ne sont ni l'appartenance à une élite internationale bilingue ni les apprentissages scolaires qui sont valorisés dans la troisième catégorie d'écoles, mais l'épanouissement de l'enfant. L'École Montessori 21 de Paris-Jaurès (EMPJ), l'École Montessori de Québec (EMQ) ou l'école Les Cotyléons de Crest, en France (ELC), mettent en avant le développement de savoirs-être :

L'EMQ participe à l'éducation de votre enfant en mettant celui-ci au centre de son attention. (...) Notre objectif est bien simple : l'émergence d'un être humain complet qui maîtrise sa liberté !"; "Autonomie, créativité, confiance en soi, respect de son environnement, collaboration, travail en projet... sont des compétences qui semblent aux parents de plus en plus nécessaires (EMPJ).

Ces écoles sont celles qui insistent le plus sur l'importance des interactions sociales, de l'entraide et de la solidarité. Ainsi, l'EMQ veut former " une personne consciente et fière de ses capacités, capable de reconnaître chez lui et chez l'autre un frère ou une sœur digne de confiance ", et l'EMPJ inclut dans son programme "un apprentissage de la gestion des émotions et des conflits pour un développement harmonieux de l'intelligence émotionnelle».

3. Mais pas du tout par les élites internationales. On le constate sur les photos, à la couleur de la peau des enfants, tous noirs par exemple dans l'école rwandaise, ou à l'utilisation des langues locales : enseignement en thaï, etc. 4. http://www.dunbarlife.com/blog/tag/preschool/, consulté le 12 juin 2017. 
Sur les photos illustrant les deux logiques d'écoles décrites plus haut, les élèves ont le regard dirigé uniquement sur l'activité scolaire qu'ils sont en train de réaliser, et leur visage, assez neutre, exprime essentiellement leur concentration. Sur les photos des écoles décrites ici, les enfants se regardent ou regardent l'adulte, et non uniquement leur travail ; leur visage est expressif (nombreux sourires, ce qui n'est pas le cas ailleurs); ils sont saisis en train de parler ou de pointer du doigt... montrant bien qu'il s'agit de privilégier, d'abord, l'échange et la communication.

Ces écoles sont implantées dans des quartiers mixtes socialement : soit dans les grandes métropoles urbaines des pays riches, d'anciens quartiers populaires dont la population s'est gentrifiée, comme le $19^{\mathrm{e}}$ arrondissement de Paris pour l'EMPJ, soit dans des zones rurales réinvesties par des populations au capital culturel important, comme l'ELC dans la vallée de la Drôme. Elles reçoivent essentiellement des classes moyennes exerçant des professions intellectuelles ou artistiques (professeurs d'université, psychologues, cinéastes, journalistes, etc.) qui consentent des sacrifices financiers importants pour y scolariser leur enfant. Ce sont les seules écoles Montessori qui affichent une volonté de mixité sociale : à l'EMPJ comme à l'ELC, les tarifs sont établis en fonction du quotient familial et peuvent varier fortement, par exemple entre $90 €$ et $790 €$ par mois à l'EMPJ ; à l'ELC, une campagne de financement participatif permet une baisse des tarifs.

Ces écoles possèdent une dernière singularité : les parents y sont

présentés comme des partenaires plutôt que comme des clients. Ils sont invités à prendre en charge certaines tâches (bricolage, ateliers) et à participer à de nombreuses réunions, ainsi qu'à des conférences éducatives organisées en soirée ou le week-end pour favoriser une cohérence entre les pratiques scolaires et familiales.

\section{Au service des plus défavorisés : les écoles Montessori des mouvements humanitaires}

Il existe d'autres écoles Montessori, très différentes de celles que nous venons d'évoquer et souvent méconnues : les écoles fondées par des mouvements humanitaires pour les enfants les plus déshérités de la planète, comme les orphelins ou les enfants des rues.

Certains programmes concernent une seule école, généralement dans le cadre d'un programme plus vaste de soutien à une communauté, comme l'École Jimma fondée dans un village reculé par la petite association Soleil d'Éthiopie (SE) et qui accueille les enfants, non scolarisés auparavant, de familles touchées par le sida. D'autres ont une ampleur plus importante, comme The Olive branch for Children (OBFC), qui a établi 28 jardins d'enfants Montessori en Tanzanie, ou la fondation Peter Hesse (FPH) qui a créé 54 écoles Montessori en Haïti et formé 750 enseignants locaux grâce à ses centres de formation. 
Ce type d'initiatives se retrouve sur tous les continents, que ce soit en Afrique (en Égypte, au Zimbabwe, en Tanzanie, en Côte d'Ivoire, au Burkina, en Éthiopie, au Ghana, en Guinée et au Congo), en Amérique latine (Pérou, Haïti), en Asie du Sud-est (Cambodge, Népal $)^{5}$... mais aussi dans les pays du Nord, comme le montre l'action d'ATD-quart monde à Noisy-le-Sec ${ }^{6}$.

Plusieurs ONG, comme OBFC et FPH, mettent en avant l'intérêt du matériel pour justifier leur choix de cette pédagogie : il peut être fabriqué localement par les communautés ${ }^{7}$; solide et réutilisable, il se révèle "économique, car il n'a pas besoin d'être remplacé souvent ${ }^{8}$. Il contraint les enseignants, de par sa présence même, à ne pas utiliser la pédagogie impositive à laquelle l'usage du pays les engage souvent. Il pallie aussi les lacunes de la formation des enseignants :

la méthode Montessori est une bonne solution pour permettre aux enseignants d'un niveau de formation assez bas de travailler d'une manière fonctionnelle en classe, car le matériel Montessori, utilisé correctement, se comprend par luimême, et les enfants peuvent en déduire des informations sans être dépendants de l'enseignant seul' .

\section{Des écoles Montessori confessionnelles : conjuguer religion et modernité}

Depuis une quinzaine d'années, les écoles confessionnelles Montessori se multiplient sur tous les continents. Selon le New-York Times Journal, quatre écoles Montessori juives religieuses ont ouvert leurs portes à Brooklyn en une décennie et il en existe au total une quarantaine aux États-Unis et plus d'une trentaine en Israël (Yee, 2014), comme l'Alef Beth Montessori School. Les écoles Montessori catholiques comme The Good shepherd Montessori school ou The good shepherd Catholic Montessori existent aux États-Unis depuis le début des années 1990, et se développent depuis peu dans d'autres pays. Ainsi, la Petite école du bon pasteur, qui a ouvert ses portes à Paris en septembre 2015, est la première de ce type en France. Il en est de même pour les écoles Montessori islamiques, comme l'Islamic Montessori school of London, implantées dans de nombreux pays du Nord comme du Sud. Au Pakistan, les jardins d'enfant

5. On trouve par exemple ici la présentation de 19 programmes sur tous les continents : Montessoriaroundtheworld. org.

6. Revue Quart monde, dossiers et documents $n^{\circ} 27$ : Les enfants acteurs de leur développement. ATD-Quart monde et l'institut supérieur Maria Montessori, regards croisés sur l'éducation. 2017.

7. The Olive branch for children évoque à ce propos «la capacité de fabriquer leurs propres matériaux à partir d'objets dans leur propre environnement ".

8. Fondation Peter Hesse.

9. Fondation Peter Hesse. Un argument qui va à l'encontre de l'approche de l'AMI, selon laquelle une formation approfondie des enseignants est nécessaire. 
Montessori, devenus très nombreux depuis leur introduction dans les années 1980, sont aujourd'hui "les écoles maternelles les plus attractives chez les habitants de Karachi », rapporte Riaz (2014). L'école Montessori islamique Brainy Bunch, ouverte en 2010 en Malaisie, a connu un succès fulgurant : en sept ans, 72 sites ont ouvert à travers tout le pays.

Ces écoles se présentent comme une réponse apportée à la demande de familles recherchant " une méthode moderne favorisant la motivation et le plaisir d'apprendre » (formule relevée sur un très grand nombre de sites), tout en assurant la transmission de leur religion et de leur culture. Le fondateur de l'école du même nom écrit ainsi :

Il y a plus d'une décennie, ma femme et moi avons rêvé de fournir aux enfants musulmans de tous âges et de tous horizons une éducation moderne de première classe, tout en assurant leur identité islamique et leur éducation. Le Brainy Bunch International Montessori, fondé en 2010, a marqué le début de la réalisation de cette vision ${ }^{10}$.

Toutes insistent sur leur volonté de concevoir un enseignement religieux qui ne soit plus fondé essentiellement sur la mémorisation ou l'obéissance à des préceptes $^{11}$, mais qui fasse appel à l'intelligence et à la sensibilité de l'enfant, dès son plus jeune âge. Elles proposent donc, à côté de "l'ambiance " Montessori classique, un matériel d'enseignement confessionnel également fondé sur la manipulation : comme par exemple des éléments de culte à échelle réduite ou des figurines de bois illustrant des paraboles ou des récits bibliques ou coraniques, permettant aux enfants, par un travail autonome basé sur le libre choix, d'intégrer des concepts religieux en touchant et en expérimentant par eux-mêmes. Le matériel spécifique d'apprentissage de la lecture et de l'écriture est adapté pour l'enseignement de l'hébreu ou de l'arabe, par exemple avec des lettres rugueuses dans ces alphabets.

La multiplication de ces écoles tient aussi à leur organisation en réseau. Dans une logique d'expansion, les écoles existantes soutiennent le développement des nouvelles structures : par exemple, la Petite école du Bon pasteur propose, durant l'été 2017, une session de formation de quatre jours destinée aux personnes souhaitant développer cette pédagogie, à un prix très bas : 170 euros pour quatre jours, incluant la pension complète en chambre individuelle ${ }^{12}$. Aux États-Unis, The National association of the catechisis of the good shepherd ${ }^{13}$ ou The Jewish Montessori society ${ }^{14}$ mettent en relation les écoles, organisent des formations et collectent des dons.

10. Traduction libre de http://www.brainybunchelementary.com/, consulté le 20 juin 2017.

11. Voir par exemple : http://www.brainybunchelementary.com/curriculum/islamic-curriculum

12. https://www.lapetiteecoledubonpasteur.com/batir-son-ecole-sur-le-roc, consulté le 20 juin 2017.

13. http://www.cgsusa.org/

14. http://jewishmontessorisociety.org 


\section{COMMENT EXPLIQUER \\ LE DÉVELOPPEMENT \\ DE CES ÉCOLES? \\ UNE ANALYSE \\ MULTI-FACTORIELLE}

\section{Un contexte favorable}

Les écoles Montessori sont d'abord portées par un contexte général : dans un grand nombre de pays de tous les continents, les pédagogies alternatives suscitent de plus en plus d'intérêt. La défiance croissante à l'égard de l'école et l'accentuation du consumérisme scolaire, chez les élites comme dans les milieux populaires (Pons et Robine, 2013), et le fait que les sociétés contemporaines mettent plus que jamais l'accent sur l'importance de la réalisation de soi et sur le développement personnel conduisent un nombre croissant de familles, dans leur recherche du " bon » établissement, à se tourner vers les pédagogies nouvelles.

La multiplication des écoles Montessori tient aussi au développement, dans un certain nombre de pays du Sud, de nouvelles classes moyennes dont la santé et l'éducation sont les priorités. Une requête avec l'outil Google trends permet de localiser les pays du monde où le terme "Montessori » a été le plus utilisé sur le moteur de recherche Google entre 2012 et 2017. Une comparaison avec une carte montrant les pays où les classes moyennes se développent le plus permet de constater que ces deux données se superposent dans plusieurs pays, notamment le Ghana, le Nigéria, l'Inde, les pays d'Asie du Sud-Est, l'Égypte, la Turquie, l'Arabie saoudite et une partie de l'Amérique latine. Ces nouvelles classes moyennes des pays émergents, en quête de la «meilleure école " pour leur enfant, sont peut-être, au niveau mondial, les premiers "demandeurs » d'écoles Montessori.

En outre, l'essor des nouvelles technologies de l'information et de la communication a permis à ces écoles de mieux se faire connaître des familles, et a contribué à leur regroupement dans des réseaux dynamiques fondés sur l'entraide, les échanges d'information, le soutien aux nouvelles structures et la co-formation, favorisant ainsi la naissance de nouvelles structures (Viaud, 2017).

\section{Réussite scolaire et bien-être : une réponse à la double demande des familles}

Dans ce contexte, la pédagogie Montessori dispose d'une particularité : elle met à la fois l'accent sur les apprentissages académiques précoces et sur le bien-être et l'autonomie des élèves. Un nombre croissant de familles recherchent des écoles attentives à l'épanouissement de leurs enfants, tout en gardant des exigences en matière d'apprentissages disciplinaires. En France, la récente 
médiatisation de Céline Alvarez ${ }^{15}$ s'est largement appuyée sur les bons résultats scolaires de ses élèves de maternelle, par exemple le fait que 13 enfants de grande section sur 14 savaient lire. "Votre enfant saura lire, écrire et compter avant les autres, et mieux que les autres " : voilà, dans ses grandes lignes, l'argument mis en avant par toutes les écoles fréquentées par les classes moyennes en ascension sociale.

La pédagogie Montessori, en promettant à la fois la réussite scolaire et l'épanouissement (bien que la hiérarchie de ces priorités varie selon le public visé, comme on l'a vu plus haut) réussit à concilier des exigences différentes, et qui pourraient sembler contradictoires.

\section{Un matériel très concret : une pédagogie immédiatement compréhensible}

Étant fondée sur l'utilisation d'un matériel très concret, cette pédagogie est immédiatement "parlante", son fonctionnement et ses finalités sont facilement compréhensibles ${ }^{16}$, pour les enfants comme pour les éducateurs ou les parents. Une analyse des discussions entre les enseignants ayant, depuis cinq ans, expérimenté les « ateliers Montessori » dans les classes maternelles de l'enseignement public français, montre que le succès rapide du matériel contribue à son adoption. De nombreux professeurs des écoles ${ }^{17}$ affirment que :

en deux ou trois séances, les enfants comprennent le principe ; leur motivation,

leur concentration, leur joie de travailler apparaissent.

Le matériel est aussi un atout, on l'a vu, pour certaines ONG dans les pays en voie de développement. Sur un tout autre plan, que cette pédagogie soit fondée sur un matériel concret permet de vendre des produits dérivés destinés à une utilisation familiale (jouets d'éveil en bois ou lettres en carton labellisés Montessori, par exemple...), servant ainsi des intérêts commerciaux qui ne sont pas innocents dans le déploiement médiatique récent de la "mode Montessori ».

\section{La moins subversive, la plus acceptable socialement des pédagogies nouvelles}

De toutes les pédagogies nouvelles, Montessori est aussi la plus acceptable socialement, à plusieurs points de vue.

15. https://www.celinealvarez.org/

16. C'est de l'impression première dont nous parlons ici : sans occulter qu'une formation approfondie est nécessaire pour maîtrise l'usage de ce matériel très particulier.

17. Viaud M.-L. : recherche en cours, 2017, ESPE Lille-Nord de France. 
Elle apparaît d'abord moins marquée politiquement que les autres. La pédagogie Freinet et la pédagogie Emilia Reggia (Dubois, 2012) ont été toutes deux liées au parti communiste dans leur histoire, ce qui les rend suspectes aux yeux d'une partie de l'opinion. La pédagogie Freinet, en outre, se donne clairement un objectif politique - former des citoyens actifs, critiques, capables de s'investir pleinement dans les décisions qui les concernent - : elle est potentiellement subversive, alors que la pédagogie Montessori est beaucoup plus neutre dans ce domaine.

Ces deux approches pédagogiques mettent aussi l'accent sur la créativité et l'expression de l'enfant (les " 100 langages », pour Reggia) bien davantage que sur les enseignements académiques, ce qui ne correspond pas aux souhaits d'une majorité de parents. En outre, les apprentissages individuels et l'attention accordée au respect des rythmes singuliers de chaque enfant ont une place centrale dans les classes Montessori, ce qui correspond à une évolution de notre société, où la prise en compte de la singularité de chacun a bien plus d'importance que l'appartenance au collectif, tandis que les enseignants Freinet, eux, se caractérisent plutôt par l'importance qu'ils donnent aux travaux et projets collectifs, à la socialisation au sein du groupe (Boncourt, 1999).

La pédagogie Montessori est, enfin, une pédagogie compatible avec l'enseignement religieux, et ce d'autant plus que Maria Montessori elle-même avait œuvré dans ce sens ${ }^{18}$, ce qui n'est envisageable ni avec la pédagogie Freinet, à l'incontestable ancrage laïc, ni avec la pédagogie Steiner, déjà fondée sur un socle spirituel spécifique.

\section{Imiter les élites pour réussir " comme eux "}

Selon une étude présentée dans le Wall Street journal du 5 avril 2011, sous le titre "The Montessori Mafia », nombre de fondateurs d'entreprises novatrices ont fréquenté des écoles Montessori et affirment y avoir acquis leur capacité à expérimenter, innover, suivre leur curiosité, comme les fondateurs de Google, de Wikipédia, d'Amazon, du jeu vidéo Les Sims... (Sims, 2011). On peut par exemple lire sur la page d'accueil de l'Alef Bet Montessori School cet article, qui a été très largement diffusé et traduit, et est cité par de nombreux sites d'écoles Montessori de tous les pays du monde:

It's simple: Israeli children need access to the kind of education that the founders of Google, Wikipedia and Amazon had. They all attended Montessori Schools ${ }^{19}$.

\footnotetext{
18. Maria Montessori a écrit plusieurs livres sur l'enseignement religieux des tout-petits comme La messe vécue pour les enfants ou L'éducation religieuse : la vie en Jésus-Christ, parus en France aux éditions Desclée de Brower, dans les années 1950.

19. "C'est simple : les enfants israéliens doivent avoir accès au type d'éducation que les fondateurs de Google, Wikipedia et Amazon ont eue. Ils ont tous fréquenté les écoles Montessori. " https://www.alefbetmontessori.org/ israel-montessori-association-ima/, consulté le 28 juin 2017.
} 
Un tee-shirt portant l'expression "The Creative Elite: the Montessori mafia » a même été mis en vente. En janvier 2016, les photos du Prince Georges, futur héritier de la couronne d'Angleterre, faisant sa première rentrée dans un jardin d'enfant Montessori, ont fait le tour du monde. Le désir d'imiter ces réussites joue incontestablement un rôle dans le succès de Montessori. Ainsi, les écoles pour classes moyennes joignent souvent le terme "élite » ou "academy" à leur nom, comme le fait l'Elite Montessori School ${ }^{20}$ : il s'agit de s'identifier aux classes supérieures.

Pourtant, dans le même temps, il n'est pas une école, ou presque, qui ne rappelle que la première Casa dei Bambini a été ouverte en 1907 pour des enfants pauvres et fut le vecteur de leur réussite. Montessori est donc, dans les représentations, à la fois une pédagogie de l'élite, mais aussi une pédagogie " accessible à tous » et adaptée aux besoins des plus défavorisés. Un article sur l'action d'ATD-Quart Monde s'est donné comme titre : "Montessori, des Beaux quartiers aux enfants pauvres de Noisy ${ }^{21}$ et la photo choisie pour l'illustrer montre des enfants de dos, en marche, sur un chemin, s'apprêtant à ouvrir une barrière. Cela peut être interprété comme révélateur d'une représentation qui pourrait s'exprimer ainsi : en offrant aux plus défavorisés la pédagogie choisie par les familles les plus aisées, on leur donne le meilleur et on leur permet de prendre un nouveau chemin.

La pédagogie Montessori est donc perçue comme pédagogie souple et adaptable, fondée sur un matériel concret qui la rend d'emblée compréhensible par les enseignants, les parents et les élèves, et compatible avec une tradition laïque comme avec un enseignement confessionnel. Son succès tient au fait qu'elle répond à des souhaits communs à tous les parents (bien-être, autonomie), mais aussi à des attentes spécifiques : les parents des classes aisées apprécient le faible taux d'encadrement, la fréquentation d'élites internationales, le bilinguisme et l'éducation au "bon goût " (classes épurées, matériel en beau bois verni, etc.) ; les classes moyennes en ascension sociale sont séduites par l'accent mis sur les apprentissages et le respect du rythme de chacun; les familles de milieux intellectuels et artistiques choisissent ces écoles pour l'épanouissement de l'enfant, l'apprentissage de la liberté et de la prise d'initiatives ; les ONG y voient l'occasion de proposer une pédagogie "élitiste " aux plus défavorisés; les écoles confessionnelles y trouvent le moyen de concilier modernité et respect des valeurs qui sont les leurs. C'est, enfin, une pédagogie vécue comme à la fois " élitiste » et "favorisant la réussite de tous".

20. Les écoles effectivement fréquentées par l'élite n'affichent jamais expressément cette caractéristique. 21. https://goo.gl/8JFZUS 
Dans un contexte où les familles sont, vis à vis de l'école, porteuses d'exigences de plus en plus nombreuses et parfois contradictoires, la pédagogie Montessori est, de toutes les pédagogies alternatives, celle qui répond le mieux à ces attentes. C'est parce qu'elle cumule ces facteurs favorisants qu'elle se développe aujourd'hui sur tous les continents, dans des contextes et auprès de publics extrêmement variés.

\section{BiblogRAPHIE}

BARTHES R. (1964) : "Rhétorique de l'image ", Communication, nº 4, p. 41-42.

DUBOIS E. (2012) : "Une utopie éducative en Émilie-Romagne", Le Télémaque 1/2012 (n 41), p. 151-161.

PONS X. et ROBINE F. (2013) : "Introduction », Revue internationale d'éducation de Sèvres, ${ }^{\circ}$ 62, p. 31-38.

RIAZ S. (2014) : New islamic schools: tradition, modernity and class in urban Pakistan, New York : Palgrave Macmillan, chapitre 5: "Knowledge at play ».

SIM P. (2011) : "The Montessori mafia » The Wall Street Journal, 5 avril 2011.

VIAUD M.-L. (2017) : "Le développement international des écoles différentes : état des lieux et perspectives ", Revue Française d'éducation comparée, n 15, juin 2017.

YEE V. (2014) : "Montessori Schools Surge in Popularity Among New Generation of Jewish Parents », 22 février 2014, New York Times, New-York edition, p. 17.

Revue Quart monde, dossiers et documents $\mathrm{n}^{\circ} 27$ (2017) : Les enfants acteurs de leur développement, ATD-Quart monde et l'institut supérieur Maria Montessori, Regards croisés sur l'éducation. 